\title{
Research on Trust Model in Distributed Network environment
}

$$
\text { Yafang Lou }{ }^{1,2, a} \text {, Yicong Zhang }{ }^{2}
$$

${ }^{1}$ College of Information Science and Technology, Peking University,100871, Beijing, China

${ }^{2}$ Department of Computer Science and Technology ZhuHai College of JiLin University, 519041, ZhuHai, China

\author{
aluckylou@sohu.com
}

Keywords: trust model, distributed, grid, robustness, reputation

\begin{abstract}
In order to study the trust model further, and solve how to establish the trust relationship between the entities, in this paper, we classify and summarize the trust model in the current distributed environment according to the different application fields, and analyze the existing trust model under the distributed environment, and study the trust model under the grid environment. In the paper, the definition of "trust" is given, and the attributes and characteristics of trust are analyzed. Finally, the deficiency of current trust model and the application of the trust model under the grid environment are concluded.
\end{abstract}

\section{Introduction}

Unlike the traditional security measures, trust is often referred to as "soft security." As the network technology is still limited, in the current research, the trust model suitable for the grid environment is not much. The trust in the different distributed environments is different, but the essence is to solve how to establish trust relationship between the entities. The following is to summarize the typical trust model in the distributed network environment, and to analyze the shortcomings of the distributed trust model and its application.Trust model is shown in Figure 1.

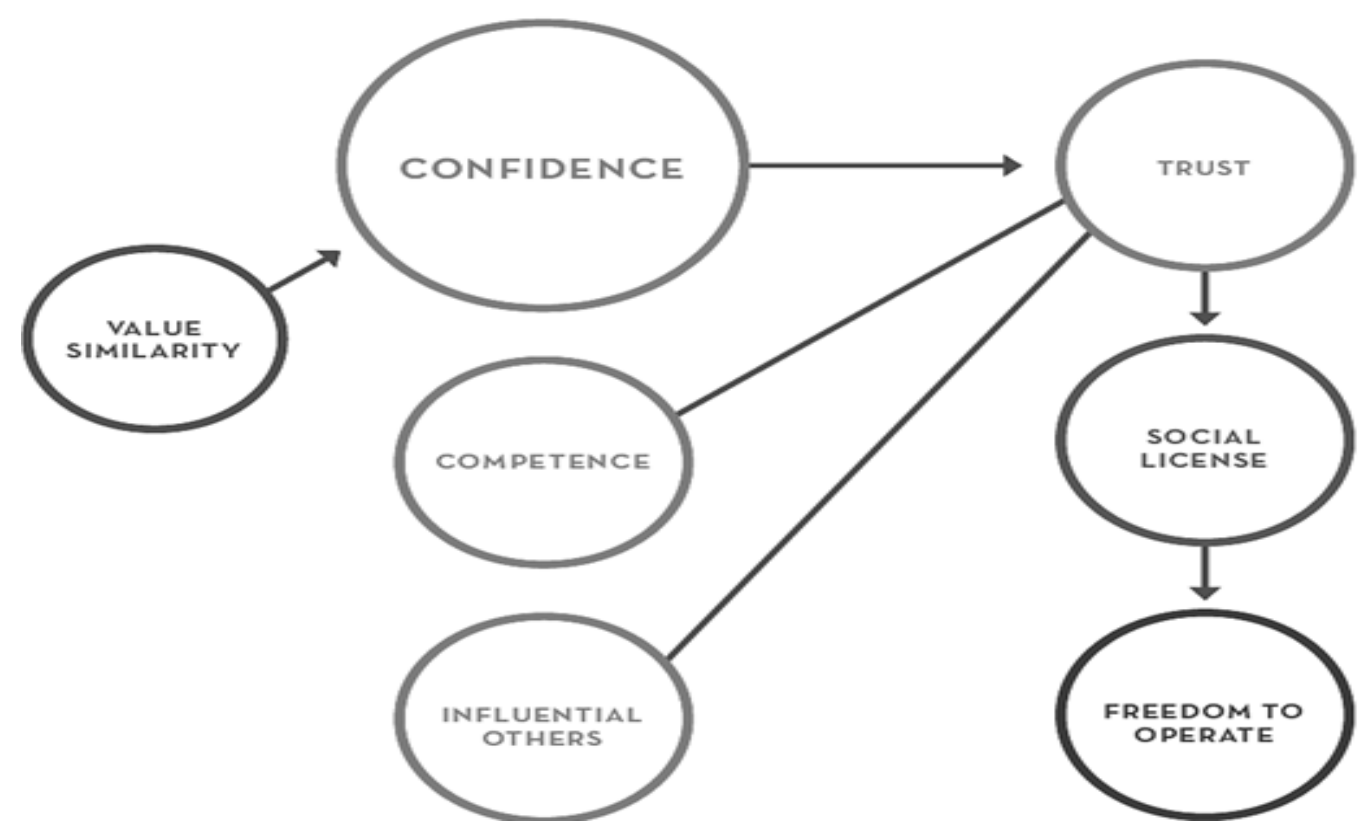

Fig1. Trust Model

\section{The distributed trust model}

\section{Eigen Trust model[1]}

The Eigen Trust algorithm, is the idea based on the delivery trust: a node $\mathrm{K}$ that give the high evaluation credibility to those nodes that provide excellent service means node $\mathrm{K}$ is likely to trust the recommendation of those nodes. The introduction of trust thought makes the Eigen Trust 
algorithm be able to construct a reputation system, and obtain the left principal eigenvector of the matrix as the global reputation value of the node by iterating the local trust value matrix normalized. The Eigen Trust model also consider the issues related to the structure of P2P systems, in addition to the area of trust management, and give a corresponding redundancy solution for this: allowing each node to calculate the global reputation for other nodes.

The experimental results show that the Eigen Trust model plays an important role in the P2P construction process.

\section{Subjective Logic Trust model}

The subjective logic opinion proposed by Jsang[2] and others introduces the concept of evidence space and opinion space to describe and measure the trust relationship, and provides a set of subjective logic for the derivation of trust and comprehensive calculation[3-4,9-10].

The main contribution of the Subjective Logic Trust model is that it provides a clear logical expression for nodes in the network how to manage their experience and the evaluation reference received[5].

\section{Damiani model}

Damiani model[6,7] achieves trust sharing with the distributed polling algorithm. Using this algorithm, the resource requester can evaluate the reliability of the resource before downloading. The algorithm is also a completely distributed algorithm. It must seek the evaluation information of the node providing resource every time to find resources, and determine which node to provide the best service through the vote of others. This voting mechanism is random and will produce too much load with each query, if the network user is huge. It brings a lot of overhead to the system. Hence, in the context of large-scale P2P system application, the algorithm is inefficient.

\section{Gupta model[8]}

The Gupta model presents a reputation system to track the user's past behavior and assist the trust evaluator to make an assessment. Gupta model is mainly applied in the P2P network with the content search and content download as the main activities. The system might lay an important foundation for the application of the credibility system in the grid environment. It promote the cooperation behavior by scoring for the good behavior while punishing the malicious . Furthermore, the system also explains how to introduce QOS into the reputation system.

\section{The lack of distributed trust mode}

In summary, the rapid development of Internet and distributed computing technology has brought great demand for trust model. From the first appearance of the concept of trust management to now, more than ten years, the research of trust model has been progressing and gradually mature: from the initial binary expression to the subjective logic, fuzzy logic and other complex forms of expression, from the simply using aggregation to integrate trust information to weighted sum, to evidence reasoning, neural networks and other complex trust information integration algorithm; from simple centralized storage and management to distributed storage and management. The research of trust model also becomes a very active research direction, same as the development of distributed computing technology. It has a wide application prospect for distributed computing environment such as grid computing, pervasive computing, P2P network and Ad hoc network. However, it must be noted that the current research of trust model still exists many deficiencies.

\section{The definition of trust and trust relationship lacks authoritative standards}

"Trust" is an important concept in the field of social sciences. " Trust relationship" is one of the most complex social relationship. The establishment and evaluation of the trust relationship is often subjectively determined by the entity. The subjectivity and uncertainty makes the definition and expression of trust and trust relation become extremely difficult. Various models have their own definitions of trust and trust relationship, but so far, the expression of trust still does not have a unified answer, which brings some difficulty to express and measure "trust".

\section{The accuracy of trust expression needs to be strengthened}

The expression of trust is the basis to evaluate trust, and its accuracy directly affects the merits of trust evaluation. The subjective uncertainty of trust, contextual relevance and other character 
increased the difficulty of expressing trust. The existing trust model is not accurate enough in the trust expression. Although there have been binary, discrete, continuous, subjective triples and fuzzy vector expression, all input source information of the expression is the subjective feedback information of the service users or consumers. It ignored the objective environmental factors such as quality of service to impact the trust evaluation. In fact, objective factors is also one of the important factors of trust evaluation. In expressing trust, we have to consider the influence of subjective and objective factors at the same time. In addition, an important feature of trust change in reality is: Entities is able to build a reliable reputation through repeat good performance, and occasionally bad behavior will quickly destroy their reputation value. It is also the case under the grid environment. This changing curve can effectively curb the malicious behavior of bad nodes and encourage the node to make honest performance. The excellent trust model should have the ability to characterize this trust and maintain the sensitivity to the changing of the entity behavior. This requires increase the punishment to the bad nodes, while the trust model characterizing the trust relationship. There still exist deficiencies for the present trust model in this respect.

\section{The robustness of the trust model needs to be further strengthened}

The Robustness of the trust model refers to the ability of the trust model to resist various malicious attacks. The attack for the trust model is divided into two categories: single-attack and complicity-attack. A single attack refers to a malicious acts initiated by a single entity against other individual or multiple entities as well as the acts of obtaining unlawful interests for themselves by fraudulent, including the dishonest acts after the high credibility accumulated by good performance and the acts to slander other nodes through the feedback information the transaction completing and so on. What complicity-attacks are more threatening to trust models mainly refer to those malicious attacks initiated by multiple nodes. These mainly include: false trading attacks that is two or more nodes to give mutually higher evaluation in the case of no real transactions in order to raise the mutual credibility, to achieve their gang's illegal purpose; the complicity defamation attack that is two or more nodes conspire to provide malicious defamation feedback information against one or more target nodes to discredit the reputation value of the target node normally occurring between competitors. In addition, the strategic attack is one of the current non-typical attack. The strategic attackers is more cunning, with the difference from the first two attacks. They will not have been cheating, sometimes performing as honest nodes to provide good service and honest feedback, sometimes performing short-term malicious attacks. It increase the difficulty to discover and enhances the hidden of cheating. The existing e-commerce trust model such as Taobao use realname strategy to curb the adverse behavior of traders. In the open P2P even service grid environment, this strategy is difficult to implement. The only solution is to improve the recognition and resistance against the malicious nodes and behavior for credibility system itself to curb the malicious behavior of the malicious nodes. The current classical trust model such as Eigen Trust model, the Peer Trust model, the Grid Eigen Trust model and so on, are still far away satisfactory and the robustness of the trust model against the malicious attacks that is various and continue to be derived.

\section{The application of the trust model in the grid environment is still rather immature}

The grid system is the distributed. Therefore, to establish the trust model in grid computing is necessary to solve the following problems: trust definition; trust attribute in specific environment; trust representation and semantics; expression and treatment of distrust and uncertainty trust; evaluation and derivation of trust relationship; efficiency and robustness of trust model; predictive ability of the trust model; trust data storage and management.

As the grid environment has its own characteristics, the most obvious features among of them are: a number of users and resources, dynamic changing; collaboration among the users; at the same time, grid users and resources generally establishing trust relationship among the autonomous regions, or integrating two levels to establish the trust relationship of a mixed level. This involves the issue of trust relationship granularity. The trust relationship is relatively simple and to describe easily if the trust model is established based on the user, but it is very unstable. The trust relationship is complicated, while it is relatively stable, if the trust relationship is established based 
on the autonomous region. At the same time, the granularity issue of the trust relationship also involves the scalability of the trust model. Furthermore, in order to better guide the entity to choose the best partner to inter act, the trust model not only be able to make an accurate assessment to the actual performance of the entity, but also accurately predict and evaluate the trend of the future behavior. The existing trust model in the evaluation of the entity's past behavior, most of the entity's past behavior to conduct a comprehensive evaluation, not the previous process of the previous conduct of the analysis, so that the entity can not make a more reasonable assessment of the past , And can not make an accurate prediction of the future behavior of the entity. The model studied in this paper does not satisfy the above problem, and can not meet the demand of trust model in grid computing. However, these trust models have made useful attempts in their respective applications, providing a method and technical reference for building a trust model on the grid.

\section{Conclusions}

Trust is a complex and blurred boundary concept, with a complex research background. This paper gives the definition of trust and analyzes the attributes and characteristics of trust after analyzing and synthesizing the previous research on the definition of trust, and describes in detail the classification of trust, expression, uncertainty representation, evaluation and derivation, and storage links. This paper makes a detailed review of the research status of distributed trust model in domestic and abroad, and analyzes the current issues in the research of trust model.

\section{References}

[1] Kamvar S D,Schlosser MT,Garcia-Molina H.The EigenTrust Algorithm for Reputation Management in P2P Networks [C],Proceedings of the 12th international conference on World Wide Web,ACM Press New York,NY,USA,2003,640-644

[2] Jsang, Ismail R, and Boy C.A survey of trust and reputation systems for online service provision [J].Decision Support Systems,2007,43(2):618-644

[3] Jsang A,The right type of trust for distributed systems,Proceedings of the 1996 New Security Paradigms Workshop.Lake Arrowhead,CA,1996[C].ACM Press,1996.

[4] Jasang A. A model for trust in security systems.Proceedings of the 2nd Nordic Workshop on Secure Computer Systems[C/0L].1997. http://security.dstc.edu.au/staff/ajosang/papers.html.

[5] Jsang A.An algebra for assessing trust in certification chains. Proceedings of the Network and Distributed System Security Symposium,NDSS 1999,San Diego, California,USA,1999[C].The Internet Society,1999.

[6] Damiani E,Vimercati D C D,Paraboschi S.A Reputation-Based Approach for Choosing Reliable Resoures in Peer-to-Peer Networks.Proceedings of the 9th ACM conference on Computer and communications security,Washington ,DC,USA,2002[C],ACM Press New York,NY,USA,207-216.

[7] Kinateder M,Rothermel K.Architecture ad Algorithms for a Distributed Reputable Servents in a P2P Network,Proceedings of the 11th international conference on World Wide Web table of contents,Honolulu,Hawaii.USA,2002[C],ACM Press,New York,NY,USA,376-386.

[8] Gupta M,Judge P,Ammar M,A Reputation System for Peer to Peer Networks.Proceedings of the 13th international workshop on Network and operating systems support for digital audio and video Monterey,CA,USA,2003[C],144-152.

[9] Jsang A,Knapskog S J. A metrtric for trusted systems[J].Global IT Security.Wien:Austrian Computer Society,1998:541-549.

[10] Jsang A.A Sujective Metric of Authentication .Proceedings of the ESRICS'98.Louvain-laNeuve,1998[C].Springer Verlag,1998,329-344. 